\title{
Cutting-edge Mathematical Tools in Processing and Analysis of Signals in Marine and Navy
}

Igor Vujovići ${ }^{1}$ Joško Šoda ${ }^{2}$, Ivica Kuzmanić3

Signal processing plays a pivotal role in information gathering and decision making. This paper presents and compares different signal processing techniques used in marine and navy applications, primarily based on using wavelets as kernel. The article covers Fourier transform, time frequency wavelet based techniques such as bandelets, contourlets, curvelets, edgelets, wedgelets, shapelets, and ridgelets. In the example section of the paper, several transform techniques are presented and commented on the harbour surveillance video stream example.

\section{KEY WORDS}

$\sim$ Harbour surveillance

$\sim$ Wavelet

$\sim$ Fourier transform

$\sim$ Signal processing

$\sim$ Edgelets

$\sim$ Curvelets
1, 3 University of Split, Faculty of Maritime Studies, Zrinsko-Frankopanska 38, 21000 Split, Croatia

E-mail: ivujovic@pfst.hr, ikuzman@pfst.hr

2 University of Split, Faculty of Electrical Engineering, Mechanical Engineering and Naval Architecture, Ruđera Boškovića 32, 21000 Split, Croatia

E-mail: jsoda@fesb.hr

\section{INTRODUCTION}

Modern electronic equipment such as GPS, radar, electronic charts, autopilot, alarm systems, automatic control systems regularly uses some sort of signal processing and analysis techniques. Signals can be divided in different sets such as stationary or non-stationary, periodic or aperiodic, deterministic or stochastic. They contain information in time, frequency and/ or any other spatial domain. Classical approach of extracting information from a signal is to transform the signal from the original domain, usually time domain, to the transformed domain, usually frequency domain. Frequency information of a given signal is usually compressed or embedded into the signal and could be extracted by applying one of the feature extraction techniques. Due to the fact that most of nowadays signals are of nonstationary and stochastic nature, and depending on the application, applying Fourier based techniques for signal analysis is not efficient. Therefore, novel mathematical tools were needed and have been proposed.

Area of image processing and analysis had rapid development in many applications in last decade - so as in the marine. Image processing and analysis play important role in the cutting-edge applications, such as in security, contra-terrorism, cargo flow, smuggling of narcotics, people (trafficking) or other goods, etc.

Among novel transforms, which are covered in the article, are wavelet-inspired transforms.

The paper is organized as follows. The Second section presents foundation of wavelet transforms chronologically, and various implementations. Also a Short Time Fourier Transform is presented as an introduction to time-frequency wavelet based techniques. The third section introduces and compares novel time frequency techniques based on wavelet kernel, such as 
edgelets, curvelets, contourlets, bandelets, wedgelets, chirplets and grouplets. Wavelet based time frequency techniques introduced in the second and third sections are applied, on real time port surveillance video stream, in the fourth section. Appropriate comments are given and there is a conclusion in the last section of the paper.

\section{WAVELET BASED TIME FREQUENCY ALGORITHMS, A BRIEF INTRODUCTION}

Fourier transform (FT) is a standard tool in many signal processing applications such as radio communications. FT is defined by (Proakis and Manolakis, 2007):

$$
F(\omega)=\int_{-\infty}^{\infty} f(t) e^{-i \omega t} d t=|F(\omega)| \cdot e^{\operatorname{arctg}(F(\omega))}
$$

where a $f(t)$ is an observed signal in the time-domain, $F(\omega)$ a spectrum of the observed signal. For a given frequency, $\omega$, equation (1) measures how much sine wave at frequency $\omega$ is comprised in the observed signal $f(t)$. The greater the value of a $|F(\omega)|$, frequency $\omega$ considerably contributes in the spectrum of observed signal $f(t)$.

However, in real applications non-stationary signals, whether periodical or non-periodical, are the most common signals obtained from measurements and systems. It is well known fact (Strang and Nquyen, 1997; Antoniou, 2006) that FT is not appropriate tool for analyzing non-stationary signals since it loses information about time domain. Therefore, new transforms, which compute time and frequency domain at the same time, are proposed.

Historically, Short Time Fourier Transform (STFT) or Windowed Fourier Transform (WFT) has been the first linear timefrequency transform proposed (Wickerhauser, 1994; Strang and Nquyen, 1997). It is defined by:

$$
\operatorname{STFT}_{f}^{\omega}(\tau, \omega)=\int_{t}[f(t) \cdot W(t-\tau)] \cdot e^{-j \omega t} d t
$$

where a STFT of a signal $f(t)$ is computed for each window centred at a $t=\tau$, at $\omega=\omega_{0}$ frequency, which is called localized spectrum. $A \cdot W(t-\tau)$ is referred to as windowing function or analysis window. An expression $e^{-j \omega t}$ is referred as FT kernel. STFT segments analyzed signal into narrow time intervals, which are narrow enough to be considered stationary on the interval. After that Fourier Transform is applied on each segment, (Gabor, 1946; Polikar, 2002).

In order to depict basis functions of time-frequency transform a time-frequency plane is proposed (Herley at. al., 1993) which is also called tiling or time-frequency tiling. The time-frequency plane of a particular basis function designates the region in the plane which contains most of that function's energy, which is shown in Figure 1.

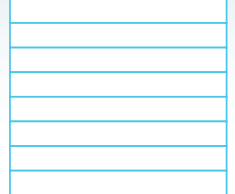

a)

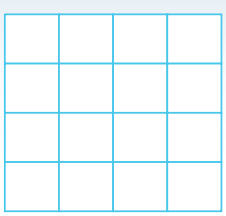

b)

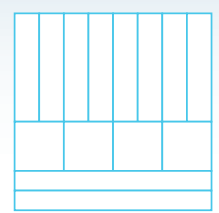

c)
Figure 1.

Tilling of the time-frequency plane for:

a) FT, b) STFT, c) wavelets.

Source: authors.

In a time-frequency plane, segments of the signal $f(t)$ have a time spread, denoted as $\Delta t$,

$\Delta t=\int_{-\infty}^{+\infty} t^{2} \cdot|f(t)|^{2} d t$

and frequency spread, denoted $\Delta \omega$

$\Delta \omega=\int_{-\infty}^{+\infty} \omega^{2} \cdot|F(\omega)|^{2} d \omega$

Then, the well-known uncertainty principle, i.e. Heisenberg principle (Wichmann, 1988), imposes the following lower bound on the product of time and frequency spreads:

$\Delta t^{2} \cdot \Delta \omega^{2} \geq \pi / 2$

Figure 1a shows time-frequency tiling for Fourier Transform. Since sine waves which are basis function for FT, have infinite compact support in time axis and ideal frequency resolution in frequency axis. Therefore horizontal strips represent timefrequency tiling. Figure $1 \mathrm{~b}$ shows time-frequency tiling for Short Time Fourier Transform. Due to the existence of window function, $W(t-\tau)$, there is a compact support in time axis and in frequency axis, therefore time-frequency tiling is presented with squares. In order to extract some features from the analyzed signal, some applications require an adaptable window function, $W(t-\tau)$, for which STFT is not suitable. In order to overcome aforementioned problem, a Wavelet Transform is proposed (Daubechies, 1992). It can be defined as follows.

Definition 2.1. Let $\psi(t) \in L_{2}(R)$ be the wavelet in the time domain and $\Psi(\omega)$ the same wavelet in the frequency domain. If and only if exists the integral:

$$
\begin{aligned}
C W T_{f}(a, b) & =\frac{1}{\sqrt{|a|}} \int_{-\infty}^{\infty} f(t) \psi *\left(\frac{t-b}{a}\right) d t= \\
& =\left\langle\psi_{a, b}(t), f(t)\right\rangle
\end{aligned}
$$


the following rules apply:

$1^{\circ} \int \psi(t) d t=\Psi(\omega=0)=0$

$2^{\circ}$ Translated (which is presented with parameter b) and scaled (which is presented with parameter a) function of $\psi(t)$ is described as $\psi_{a, b}(t)=\frac{1}{\sqrt{|a|}} \cdot \psi\left(\frac{t-b}{a}\right)$ where $a, b \in R$ and $a \neq 0$. Function $\psi(t)$ is called mother wavelet, and $\psi\left(\frac{t-b}{a}\right)$ is

dilated version of mother wavelet at given scale $a$. In practical applications, scale parameter is always $a \leq 1$.

$3^{\circ}$ Normalization rules apply: $\left\|\psi_{a, b}(t)\right\|=\|\psi(t)\|$ and

$4^{\circ}$ Then the CWT satisfies the sufficient and necessary conditions and it is called Continuous Wavelet Transform (CWT) (Kingsbury and Magarey, 1997).

Wavelets $\psi(t)$ can be considered as band pass filters (Vetterli and Kovačević, 1995; Rioul and Vetterli, 1991).

Figure 1c shows time-frequency tiling for Continuous Wavelet Transform. Since wavelets are compactly supported in both axes, an adaptable window exists in time-frequency plane. At high frequencies there is good localization in time, and at low frequencies there is good localization in frequency (Mallat, 1999). It has to be pointed out that the area of time-frequency window is always constant so Heisenberg's uncertainty principle is preserved.

In order to use Continuous Wavelet Transform with computer and in order to ensure convergence of CWT algorithm, sampling of coefficients is needed (Kingsbury and Magarey, 1997). An algorithm obtained by sampling coefficients of CWT transform is known as Discretized Continuous Wavelet Transform (Christopher and Walnut, 2006).

If the wavelet equation $\psi_{a, b}(t)=\frac{1}{\sqrt{|a|}} \cdot \psi\left(\frac{t-b}{a}\right)$ is discretized by choosing coefficients of the scale, $a$, and the translation, b, to form a dyadic pair, (Vetterli and Kovačević, 1995), a discretized version of wavelet equation is obtained:

$\psi_{m, n}(t)=2^{-m / 2} \cdot \psi\left(2^{-m / 2} \cdot t-n\right)$

Then eq. (6) becomes:

$$
\begin{aligned}
& c_{m, n}=|2|^{-m / 2} \int_{-\infty}^{\infty} f(t) \cdot \psi^{*}\left(2^{-m / 2} \cdot t-n\right) d t= \\
& =\left\langle f(t), \psi_{m, n}(t)\right\rangle
\end{aligned}
$$

which is known as Discretized Continuous Wavelet Transform. It is well known that in practical applications, function $f(t)$, from Eq. (6), cannot be described analytically, therefore solving an

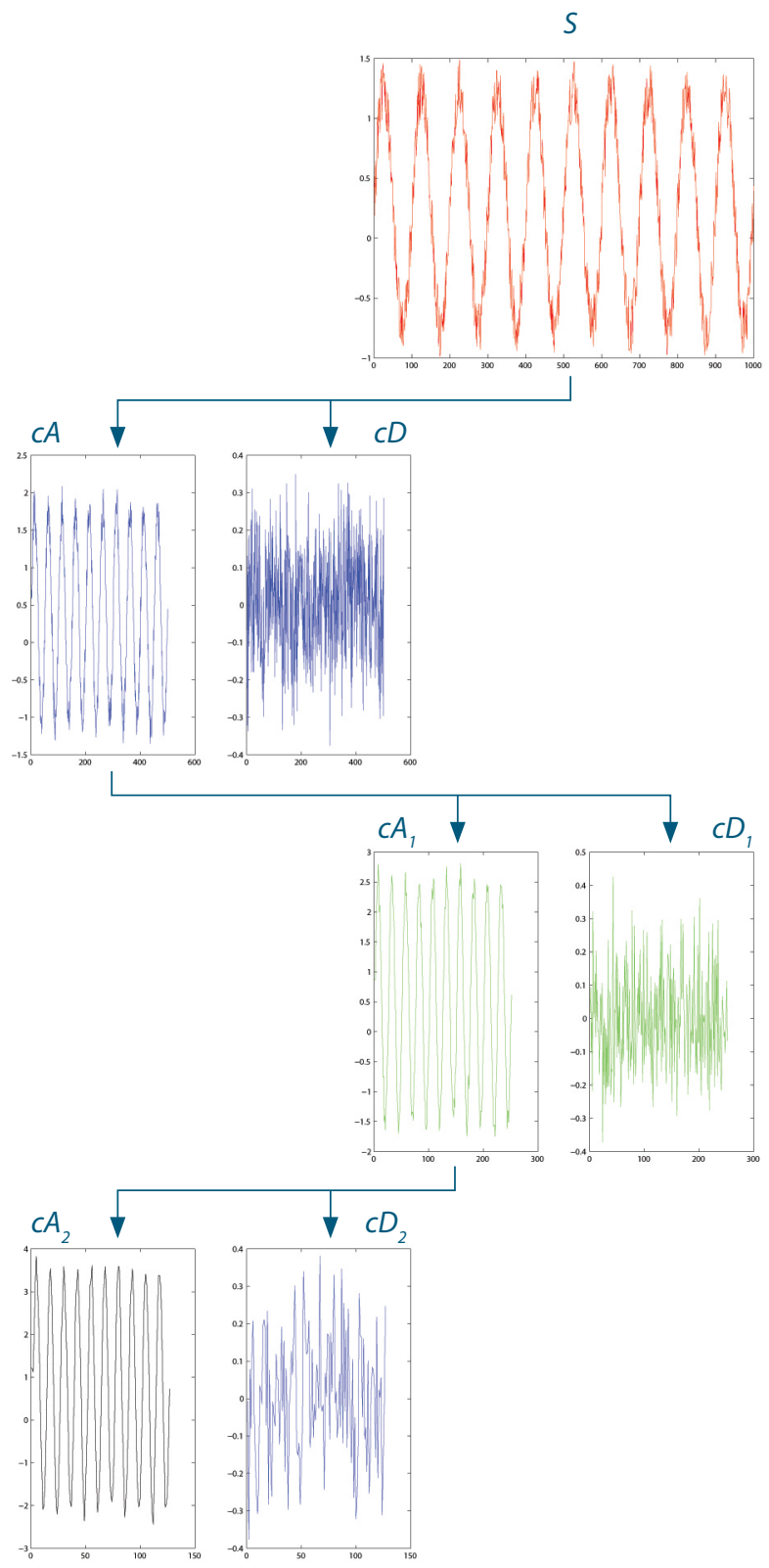

Figure 2.

Wavelet decomposition of the signal (Misiti et al, 1997). Source: authors.

integral (6) is time consuming at best, or even impossible. In order to overcome aforementioned problem a new transform is proposed, Discrete Wavelet Transform (DWT).

DWT overcomes problem of computing CWT coefficients, and it is suitable in fast computer algorithms. Moreover, since DWT algorithm converges rapidly, it is suitable for real time applications. 
Historically, two groups of authors, in late $20^{\text {th }}$ Century, proposed techniques for computing DWT coefficients. First group of authors (Vetterli and Gall, 1989) proposed Multiresolution Signal Analysis (MRA) technique or pyramidal algorithm. Second group (Crochiere et al., 1975; Crochiere and Sambur, 1977) proposed subband coding algorithm. Pyramidal algorithm is especially suitable and widely used in image processing applications (Mallat, 2009). Relationship between MRA and wavelet analysis are investigated and presented by Mallat (Mallat, 2009) and Daubechies (Daubechies, 1992).

Foundations of Discrete Wavelet Transform are based on Multiresolution Analysis and Filter Bank Theory (Strang and Nquyen, 1997).

A subband coding algorithm is the fast algorithm that computes discrete wavelet coefficients, and it is based on filter banks.

In order to implement filter bank theory with MRA introduction of a scaling function, $\phi(t)$, is proposed (Strang and Nquyen, 1997). Scaling function, $\phi(t)$, is defined as follows:

$\phi(t)=\sum_{k=0}^{N} 2 \cdot h(n) \cdot \phi(2 t-n)$

where a $h(n)$ are coefficients of a low pass half band filter. Equation (9) is known as dilation equation (Vetterli and Kovačić, 1995). Relationship between wavelet equation and FB theory is given with the following equation:

$\psi(t)=\sum_{k=0} 2 \cdot d(n) \cdot \phi(2 t-n)$ where $\mathrm{ad}(\mathrm{n})$ is coefficients of a high pass half band filter. Equation (10) is known as wavelet equation (Vetterli and Kovačić, 1995).

Figure 1 shows decomposition of a signal, $\mathrm{S}$, at three levels, applying subband coding algorithm (Misiti et al., 1997). After a signal $S$ is passed through the filter bank, which consists of two filters, half band low pass filter and half band high pass filter, and then applying downsampling procedure, a coefficients of approximation, CA1, and a coefficients of details, cD1, are obtained, at the first level of decomposition. It has to be pointed out that with applying aforementioned procedure, coefficients $\mathrm{CA} 1$ and $\mathrm{CD} 2$ are halved with respect of total number of coefficients of analyzed signal S. At the second level of decomposition, approximation coefficients $\mathrm{CA} 1$, are passed through filter bank and then downsampled, which produces approximation and details coefficients, CA2 and CD2. CA2 and CD2 coefficients contain exactly half of the total number of $\mathrm{CA} 1$. At the last level of decomposition, CA1 coefficients are passed through filter bank and then downsampled, which produces $C A 3$ and CD3 coefficients at the third level. As previously, coefficients $C A 3$ and $C D 3$ contain half of the total number of $C A 2$ coefficients. Wavelet decomposition algorithm is also known as logarithmic signal decomposition algorithm (Christopher and Walnut, 2006).

Described procedure has one potential drawback, in order to be able to assemble algorithm, you need to have an extensive knowledge about Fourier analysis and filter bank theory, since all programming is required to be done in frequency domain. In order to avoid frequency domain, Wim Sweldens proposed Second Generation Wavelet, SGW (Sweldens, 1998). His algorithm

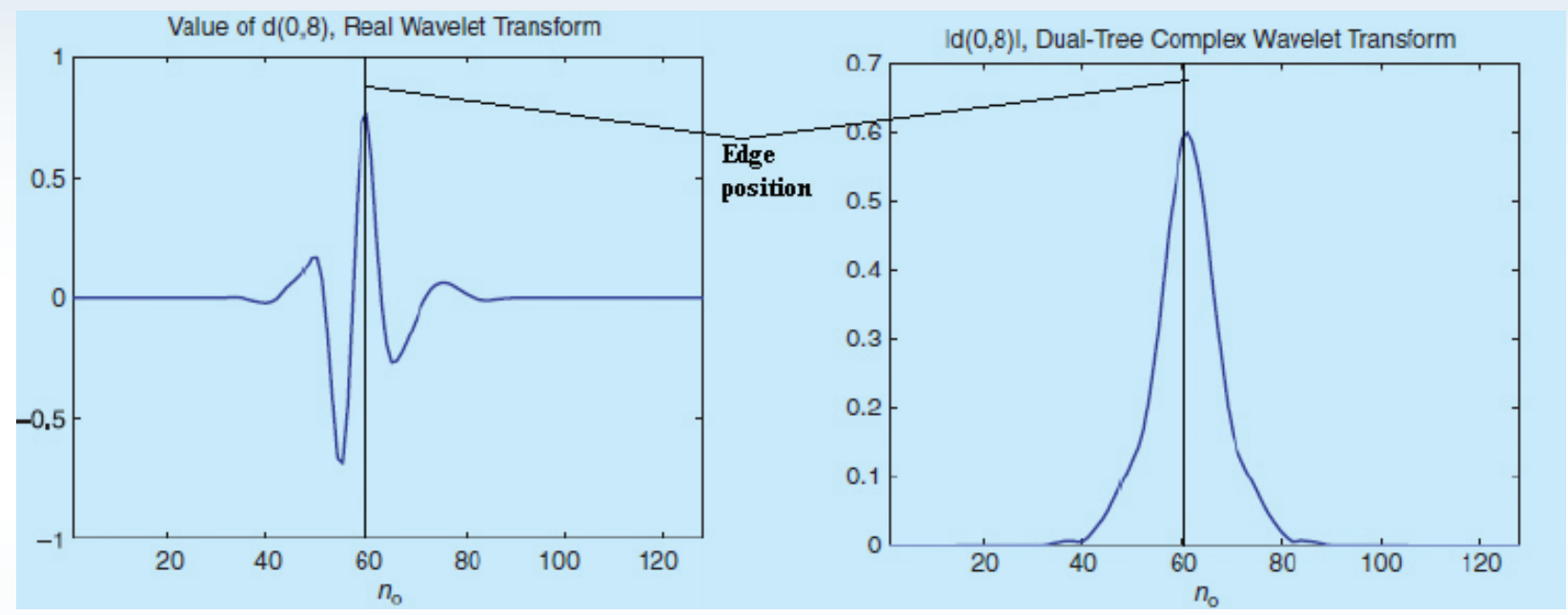

a)

b)

Figure 3.

Edge detection by: a) DWT, b) dual-tree CWT.

Source: authors. 
is programmed entirely in time domain and is known as lifting scheme (Jansen and Oonincx, 2005).

DWT exhibits four main flaws: oscillations, shift variance, aliasing and lack of directionality which are especially emphasized in image processing. It is well known that wavelet functions have zeroed mean. Therefore, the shape of the wavelet function is oscillating and wavelet coefficients can gain either positive or negative value. By applying the DWT results in weak edge definitions of the observed signal, and smear edge effect could be produced, in discontinuity localization. Figure 3 illustrates the edge detection by applying DWT and CWT transform in the same image. Figure 3a shows position of DWT wavelet on the edge of an image. Since wavelet has zeroed mean it oscillates on the edge, and results in smear edge effect which is presented in the analysis image. Figure $3 \mathrm{~b}$ shows position of CWT wavelet on the edge of an image. Since the coefficients of CWT are only positive, edges of the image are more emphasized.

Shift variance presents the problem in interpretation of the signal since it greatly perturbs the wavelet coefficient oscillation pattern around singularities. Shift variance also complicates wavelet-domain processing since algorithms must be made capable of coping with wide range of possible wavelet coefficient patterns caused by shifted singularities (Selesnick et al., 2005).

When DWT transform is applied, at each level of signal decomposition, coefficients are computed by applying non-ideal low-pass and high-pass filters and then they are downsampled, which results in substantial aliasing. Since in real application some of the thresholding, filtering or quantization follows, it is impossible to obtain perfect reconstruction, which leads to artefacts in the reconstructed signal. Lack of directionality is also presented in higher dimensions. While Fourier bases functions are highly directional plane waves, a wavelet function lacks directionality. Non-directionality presents a problem in advance image processing and analysis applications, such as finding edges and ridges. It has to be pointed out that Fourier basis does not suffer from the same problems as wavelets.

To overcome mentioned DWT problems, and with Fourier transform as guidance, a complex wavelet are proposed (Selesnick et al., 2005), denoted $\psi_{c}(t)$ :

$\psi_{c}(t)=\psi_{r}(t)+j+\psi_{i}(t)$

where a $\psi_{r}(t)$ is an even function, a $\psi_{i}(t)$ an odd function. A $\psi_{r}(t)$ and a $\psi_{i}(t)$ are phase shifted by $90^{\circ}$ and make so called Hilbert transformation pair. The necessary condition is that a $\psi_{c}(t)$ is an analytical function. It can be shown that the large signal magnitudes correspond with the existence of the discontinuity in the analyzed signal and sudden phase changes correspond with the position of the discontinuity.

Complex wavelets are proposed in order to comply with more and more demanding applications. Complex Wavelet
Transform (CWT) is built on the DWT basis (Selesnick et al., 2005). First papers which deal with CWT were published in 1995. (Gagnon, Lina and Goulard, 1995; Lina and Gagnon, 1995). Algorithm for CWT calculation is introduced in 1997 (Kingsbury and Magarey, 1997; Kingsbury, 1998), and it is known as DualTree Complex Wavelet Transform algorithm.

CWT is primarily used in image processing applications, radar, speech, music, and multidimensional signals applications.

Wavelets are also widely used in navy coding of the secret data. They are also used in coding the video information in Internet transmission standards, video coding, digital imaging, etc. Due to increasing use of communication and multimedia in modern ships, all the areas of application can be found in various ships.

\subsection{Multidimensional wavelet transform}

All transforms mentioned in previous section are one-dimensional. In order to implement transforms for multidimensional signals, such as images, one must extend onedimensional theory to multidimensional case. This generalization is not always as simple as it may seem. Implementation of the DWT to multidimensional case requires separable wavelet bases in all dimensions.

In wavelet theory, it is usual to operate with time and frequency. However, physical interpretation can be different. The axis which denotes time can be replaced with some other axis. The frequency axis can also be replaced with the other axis. The mathematical properties of the transform remain the same. In image analysis, meanings of the axis are colour, number of pixels and coordinates of the pixel with denoted colour. Multidimensional transform involves causality problems. Therefore, a trick is used: one-dimensional algorithm is extended to more general case by separable approach and use of tensors.

Application of DWT to the image implies processing of rows and columns separately. Firstly, rows are filtered by LP and HP filters, then downsampling is performed. After that, the same is performed with columns. This process is shown in Figure 4. Final results are coefficients of two-dimensional wavelet transform. An $A_{k+1}$ is an approximation obtained by applying low pass filtering of both the rows and columns, i.e. LL output. Combinations of low and high and high and low filtering result in coefficients of details, i.e. LH and HL coefficients, which are known as horizontal and vertical coefficients. Finally, if both rows and columns are filtered with HP filter, then diagonal details are obtained.

One level of the reconstruction can be described with equation: 


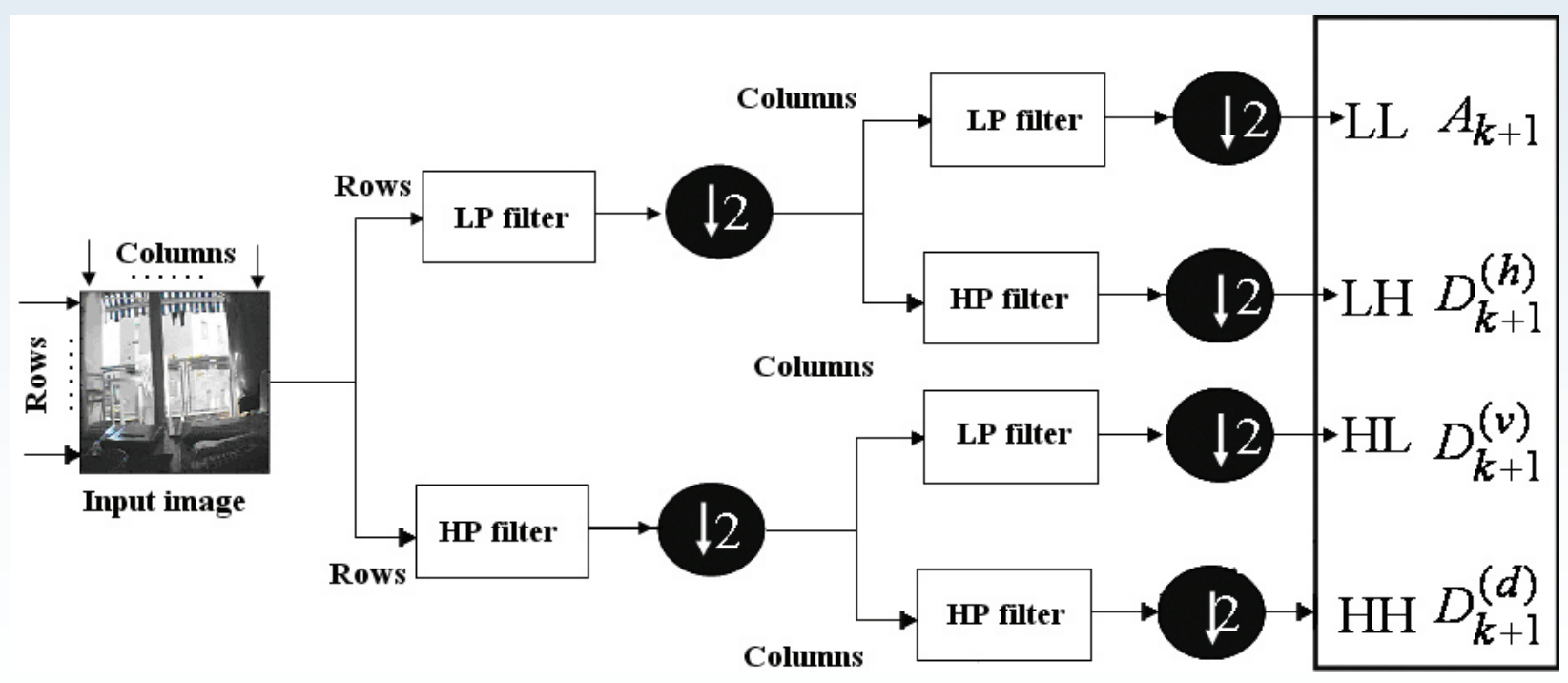

a)

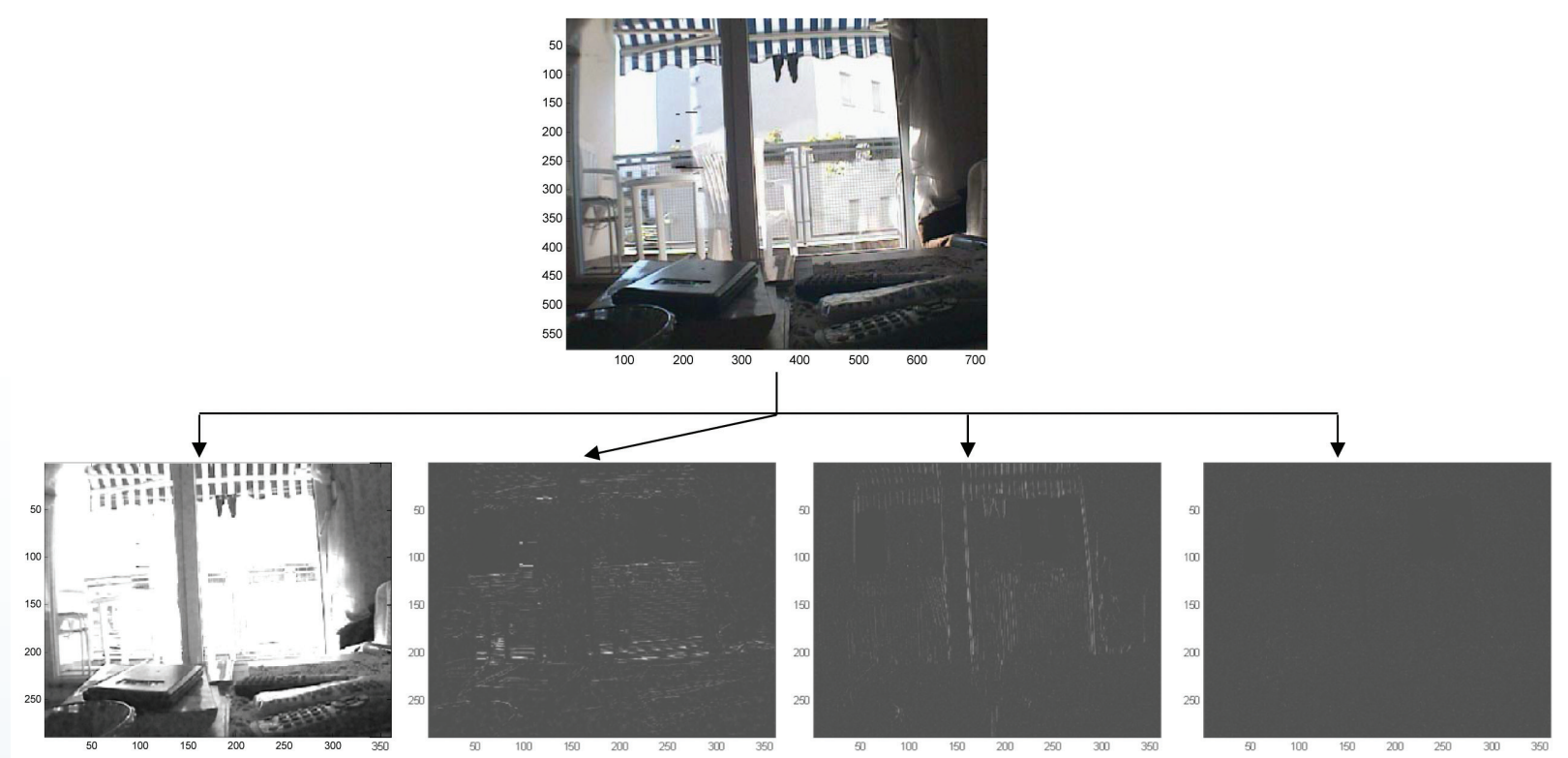

b)

Figure 4.

Wavelet analysis of the image: a) scheme (Polikar, 2002), b) an example. Source: authors. 


$$
\begin{array}{r}
f_{k}(x, y)=\sum_{n=-\infty}^{\infty} \sum_{p=-\infty}^{\infty}\left(A_{k+1}(n, p) \cdot \phi(x-n) \cdot \phi(x-n)\right. \\
+D_{k+1}^{h}(n, p) \cdot \phi(x-n) \cdot \psi(x-n) \\
+D_{k+1}^{v}(n, p) \cdot \psi(x-n) \cdot \phi(x-n) \\
\left.+D_{k+1}^{d}(n, p) \cdot \psi(x-n) \cdot \psi(x-n)\right)
\end{array}
$$

where a $\phi$ is the scaling function and a $\psi$ the wavelet function.

Nowadays applications operate mostly with video streams or images. Due to increased need for image processing algorithms, novel transforms are mostly used in multidimensional case.

\section{NOVEL WAVELET-INSPIRED TRANSFORMS}

Wavelets have exhibited a huge success in one-dimensional problems of signal processing and analysis. Furthermore, wavelets have a great success in image compression tasks, for example as in $\mathrm{FBI}$ application for fingertip database storage. In images consisting domains of smoothly varying grey values, separated by smooth boundaries, DWT exhibits problems that are visible in the form of compression artefacts. The problem is visible in the form of compression artefacts. Tensor-product construction (used in 2D-WT) is not flexible enough, causing the inability to repeat results obtained in 1D case (Führ et al., 2006). In order to further improve and enhance analysis, different transforms are proposed.

The phenomena of interest occur often along curves and sheets. The examples are the edges in the image. However, wavelets are suited for dealing with e.g. singularities, but there are ill-suited for detecting and dealing with intermediate dimensional structures.

\subsection{Bandelets}

Bandelets are constructed from an orthonormal basis that is adapted to geometric boundaries (Pennec and Mallat, 2005). Bandelets can be constructed and regarded as a warped wavelet basis. They are used to transform smooth functions on smoothly bounded domains. Since many other transforms utilize wavelets as well, they are called wavelet-inspired novel transforms. Contourlets and curvelets take account of geometric structure, but they utilize wavelets as well (Peyre et al., 2007). All novel transforms start with wavelet basis. The point is in changing the coordinate system and/or rotating the basis.

Proposition 3.1. Bandeletization of wavelet coefficients using an Alpert transform defines a set of bandelet coefficients if and only if a vector corresponding to a sampling of a function with an anisotropic regularity is well approximated with a few vectors from the Alpert basis. These coefficients can be written as inner products $\left\langle f, b_{j, \ell, n}^{k}\right\rangle$ of the original image $f$ with bandlet functions that are linear combinations of wavelet functions:

$b_{j, \ell, n}^{k}(x)=\sum_{p} a_{\ell, n}[p] \psi_{j, p}^{k}(x)$ where an $a_{l, n}[p]$ is the coefficient of the Alpert transform.

\subsection{Curvelet transform}

The curvelet transform is a multiscale pyramid with many directions and positions at each length scale, and needleshaped elements at fine scales (Candés et al., 2006). Scaling part of the curvelets obey a parabolic law. Namely, at scale $2^{-j}$ , each element has an envelope which is aligned along a ridge of length $2^{-j / 2}$ and width $2^{-j}$. Curvelets provide optimally sparse representation of objects with edges or wave propagators. There also have micro local features that help in reconstruction of severely ill-posed problems.

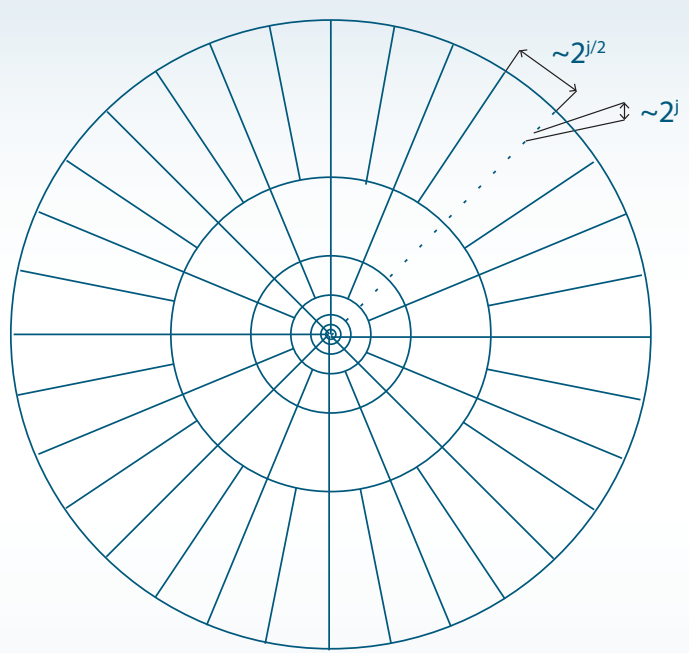

Figure 5.

Curvelet induced tilling of the frequency plane (Candés et $\mathrm{al}, 2006)$.

Source: authors.

Definition 3.1. (Candés et al., 2006) Let $x=\left(x_{1}, x_{2}\right)$ be random variable. Curvelet is defined at scale $2^{-j}$ orientation $\theta_{1}$ and position $x_{k}^{(j, l)}=R_{\theta l}^{-1}\left(k_{1} \cdot 2^{-j}, k_{2} \cdot 2^{-j / 2}\right)$ by:

$\varphi_{j, k, l}(x)=\varphi_{j}\left(R_{\theta \ell}\left(x-x_{k}^{(j, \ell)}\right)\right)$

where a $R_{\theta}$ is the rotation by $\theta$ radians and it is mathematically Givens rotation (Golub et al., 1996):

$R_{\theta}=\left[\begin{array}{cc}\cos \theta & \sin \theta \\ -\sin \theta & \cos \theta\end{array}\right]$

The most important properties of the curvelet transform are (Candés et al., 2006): tight frames, parabolic scaling, oscillatory 
behaviour and vanishing moments.

The polar dyadic parabolic partition of the frequency plane is the bases of the curvelets construction. If different tilling is used, other transforms are obtained. For example (Candés et al., 2006):

a) A directional wavelet transform is obtained if we divide the corona into a constant number regardless of scale.

b) A ridgelet transform is obtained by subdividing each dyadic corona into $C \cdot 2^{\mathrm{j}}$ angles.

c) A Gabor analysis is obtained if we substitute coronae with fixed width for dyadic coronae.

Furthermore, an adaptive partitioning of the frequency plane can be created, which best matches the features of the analyzed signal. This is the construction of so called "ridgelet packets".

\subsection{Contourlets}

The contourlet transform is a geometrical image based transform. In this transform, the Laplacian Pyramid is firstly used to capture the point discontinuities, then it is followed by a Directional Filter Bank to link point discontinuities into linear structures. The Laplacian Pyramid is used to decompose an image into a number of radial subbands. The Directional Filter Bank is used to decompose each radial subband into a number of directional subbands (Anaraki et al., 2007).

\subsection{Shapelets, Ridgelets, Edgelets and other transforms}

Proposition 3.2. The shapelet decomposition enables an approximation calculation of the 2D object by a finite series (Melchior et al., 2007):

$I(\vec{x})=\sum_{n_{1}, n_{2}}^{n_{1}+n_{2}=n_{\max }} I_{\vec{n}} B_{\vec{n}}\left(\vec{x}-\vec{x}_{c} ; \beta\right)$

where a $\bar{x}=\left(x_{1}, x_{2}\right)$, a $\vec{n}=\left(n_{1}, n_{2}\right)$, and the shapelets basis function is expressed by (Refregier, 2003):

$B_{n}(x ; \beta)=\beta^{-1} \phi_{n 1}\left(\beta^{-1} x_{1}\right) \phi_{n 2}\left(\beta^{-1} x_{2}\right)$

The basis is related to the 1D Gauss-Hermite polynomials with:

$$
\phi_{n}(x)=\left[2^{n} \pi^{0,5} n !\right]^{-0,5} H_{n}(x) e^{-0,5 x^{2}}
$$

where $\mathrm{a} \mathrm{H}_{n}(\mathrm{x})$ is a Hermite polynomial of an order $n$.

The wavelet-inspired transforms use the wavelet basis that is a dimensional generalization of the wavelet transform designed to represent images at different scales and different angles. The ridgelet transform uses the Finite Radon Transform (FRAT) as a basic building block.

Wedgelets were proposed in 1999 (Donoho, 1999; Donoho et al., 1999). The purpose of proposed transform was approximation of the piecewise constant images with smooth boundaries.

Proposition 3.3. Let $Q_{j}$ be the set of dyadic squares of size $2^{-j}$ expressed by:

$Q_{j}=\left\{\left[2^{-j} k: 2^{-j}(k+1)\right] \times\left[2^{-j} \ell: 2^{-j}(\ell+1)\right]\right\}$

with $0 \leq k, I \leq 2^{j}$. The set is the union:

$Q=\bigcup_{j=0}^{\infty} Q_{j}$

Dyadic portion of the image is given by the tilling $Q$ defined in the domain $[0,1]^{2}$ of dyadic squares of arbitrary size. If $Q$ is defined as above, a wedgelet tilling is obtained by splitting each element $q \in Q$ into at most two wedges along a suitable straight line.

An edgelet is a short segment of line or curve $(\mathrm{Wu}$ and Nevatia, 2005).

Proposition 3.4. The affinity between the edgelet and the image I at location $w$ can be calculated by the expression:

$$
S(w)=\frac{1}{k} \sum_{i=1}^{k} M^{\prime}\left(u_{i}+w\right)\left|\left\langle n^{\prime}\left(u_{i}+w\right), n_{i}^{E}\right\rangle\right|
$$

where $\left\{u_{i}\right\}_{i=1}^{k}$ denote the location of points in the edgelet, $\left\{n_{l}^{E}\right\}_{i=1}^{k}$ normal vectors at those points, a $k$ the length of the edgelet, $a$ I the input image, which have $\mathrm{M}(p)$ as edge intensity and $n(p)$ normal vector at location $p$ of the image $I$.

Note: Since edge intensity and normal vector are unknown, it is impossible to solve proposition 3.4. Therefore, the mentioned unknowns must be calculated by one of the edge detectors. In (Wu and Nevatia, 2005) the problem is solved by Sobel kernel of size $3 \times 3$. However, we submit that Canny or some wavelet detector can produce better results. If same wavelets are used to generate an edgelet and an edge detector, time of execution can be reduced.

\section{APPLYING WAVELET BASED TIME FREQUENCY TECHNIQUES ON PORT SURVEILLANCE VIDEO STREAM EXAMPLE}

Image processing and analysis play an increasing role in the security of ports due to increasing threats in the marine transport. Therefore, example of the image as the signal is taken deliberately. The chosen image shows the high traffic of small vessels, which can be the potential problem. Camera can detect suspicious movement, i.e. terrorist attack or fire aboard.

Figure 6 shows an arbitrarily chosen image from the port surveillance camera. It is the JPG file of the size $2592 \times 1944$. The colour space is RGB. It is analyzed by: 


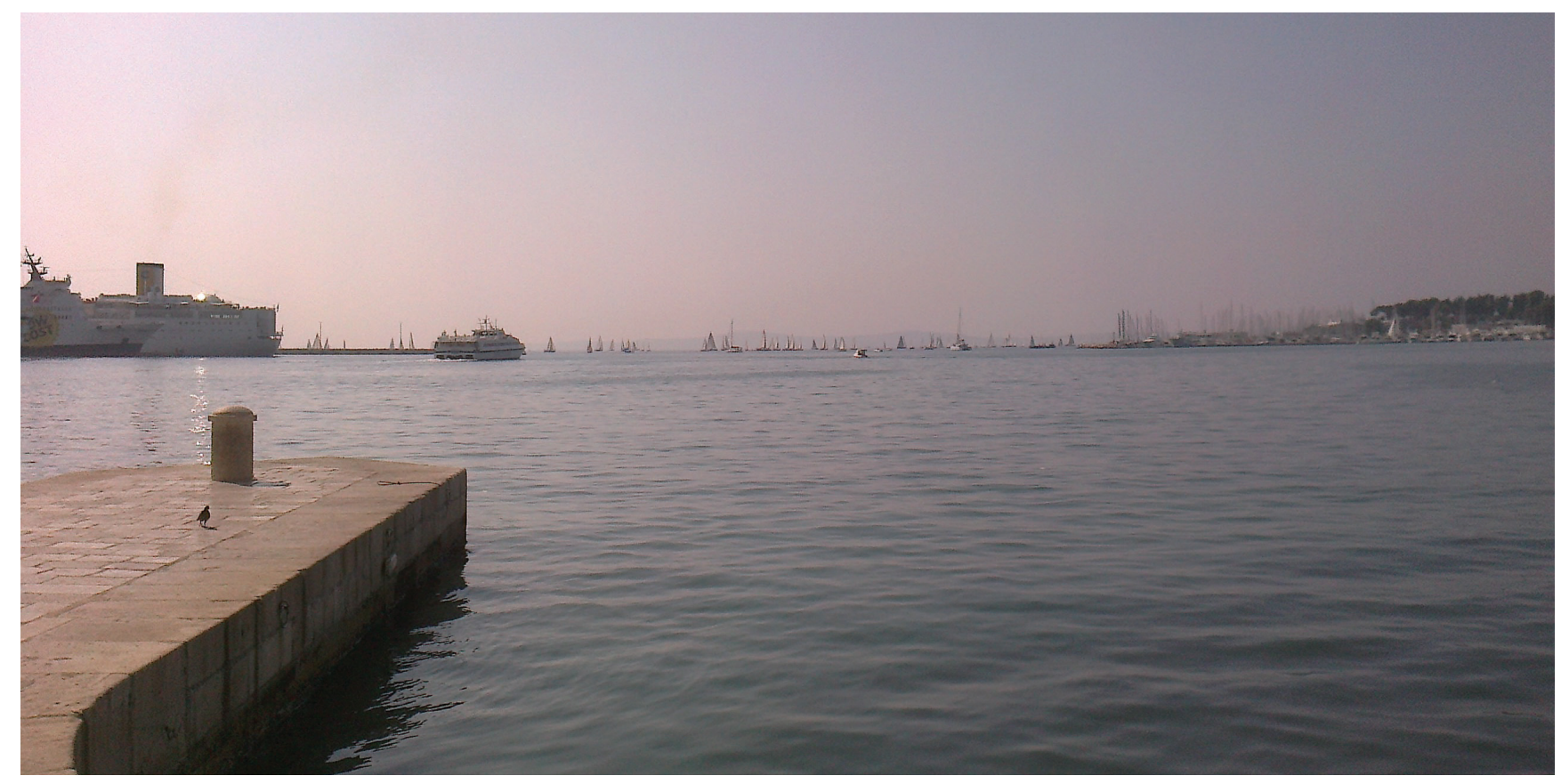

Figure 6.

The original arbitrarily chosen image from the port surveillance camera.

Source: authors.
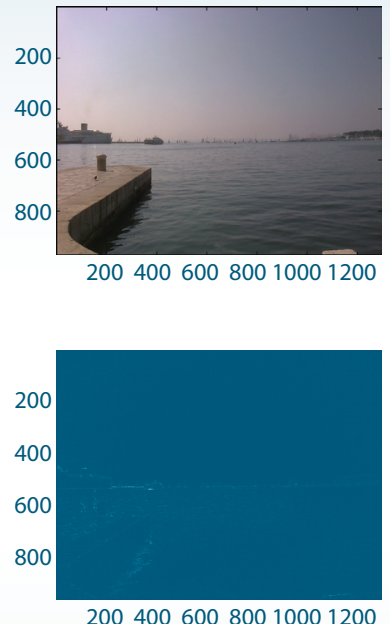

Figure 7.

Wavelet decomposition of arbitrarily chosen image obtained by the port surveillance camera.

Source: authors.
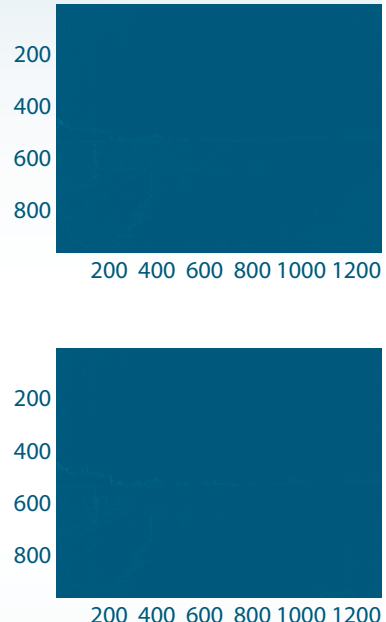

20040060080010001200

Figure 8

Conturelet coefficients at two levels of decomposition. Source: authors.

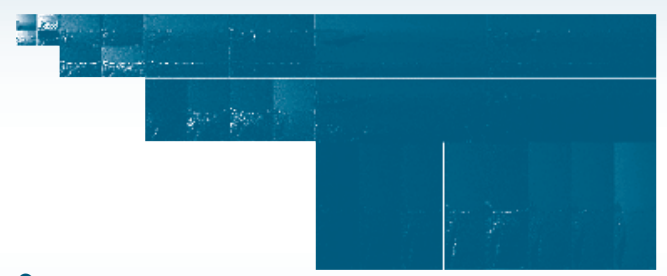

Figure 9.

Contourlet coefficients at three levels of decomposition. Source: authors. 


\section{- DWT \\ - contourlet and \\ - curvelet transform.}

Figure 7 shows an example of the wavelet decomposition at the first level. The upper left part of the image shows approximation coefficients. Horizontal details are shown in upper right part of the image. Due to small values of the coefficients, it would appear black. To be visible, we multiplied it by factor 8 in comparison to the approximation coefficients. The lower left part of the image shows vertical details and lower right diagonal details. All details are multiplied by 8 to be visible. It can be observed that the wavelet approximation takes all the energy contained in the image signal (the brightest image). The details exhibit chaotic behaviour at the sea level, and regular smoothness at the dock.

The wavelet produced coefficients are too scattered and do not show the direction of possible movement. Moreover, the wavelet coefficients do not emphasize the motion of any kind.

It can be calculated that the size of the sum of DWT coefficients and original image are the same. Therefore, any gains can be obtained only by reducing the coefficients by thresholding or the exclusion of the coefficients which are redundant.

Figures 8 and 9 show the results of the contourlet decomposition at two and three levels. The input image is shown in Figure 6 . It can be seen that contourlet transform emphasizes the sharp edges.

However, the grain structure of the image prevents higher vision applications to segment the image correctly. In favour of the contourlets, it should be noted that the example is arbitrary in choosing contourlet as well. It is possible that other contourlet would produce better results.

Figure 10 shows the analysis of the Figure 6 by applying Fast Discrete Curvelet Transform via wedge wrapping. Curvelets can be real or complex-valued. In the presented example, the complex-valued curvelets are chosen. Regarding the software application, possibilities for the coefficients at the finest level are curvelets and wavelets. Curvelets are selected as the example shown in the image.

Upper right image is the curvelet approximation of the Figure 6 . Note that the 8 details (by different angle of curvelet basis) should look black. They are multiplied by 2048 times in order to be visible by the human eyes in offprint.

It can be observed that the size of the details is rotating
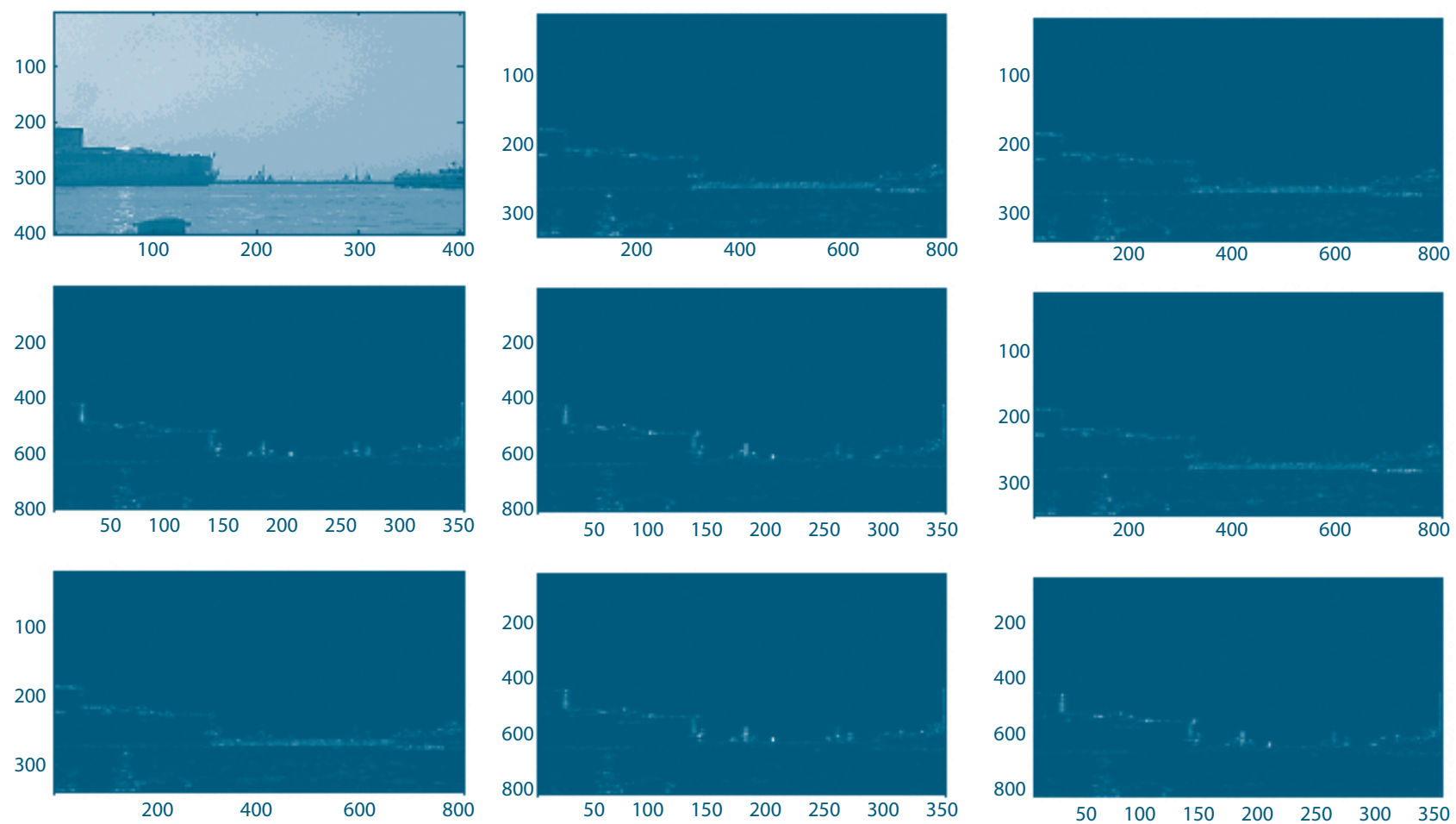

Figure 10.

Curvelet transform example.

Source: authors. 


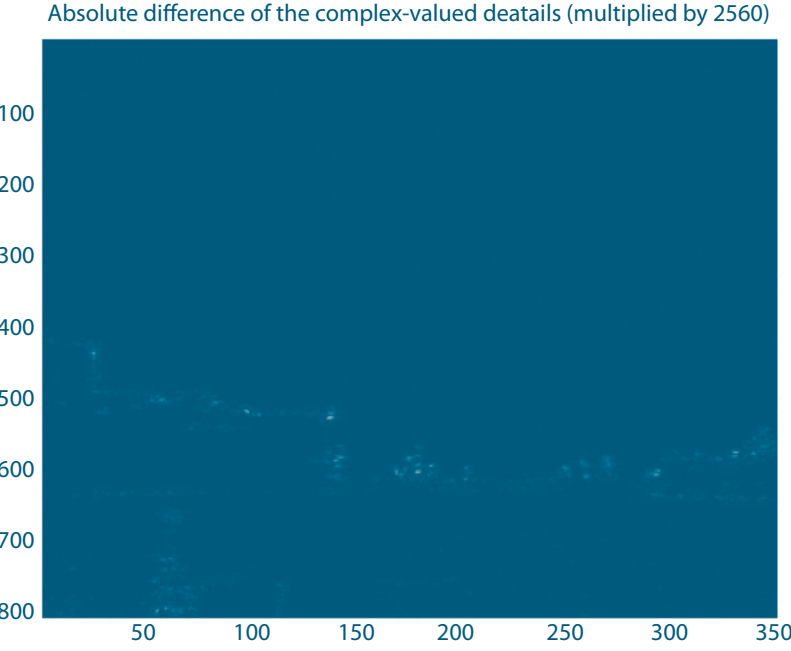

Figure 11.

Absolute difference of the details.

Source: authors.

and that approximation has different size. It is the opposite to the wavelet transforms, which all have the same sizes (both approximation and details).

Figure 11 shows the absolute difference of the details multiplied in order to be visible.

\section{CONCLUSIONS}

The cutting-edge mathematical tools for signal processing and analysis can be divided into two groups:

- adaptive geometry-based tools such as wedgelets and related constructions and

- directional frames, such as curvelets or ridgelets, to name but a few.

Depending on application, a researcher has to be able to choose appropriate wavelet based transform algorithm.

All presented techniques, however advanced, are not applicable in every instance. Lot of care has to be taken in order to choose the right algorithm. Some techniques are better suited for the signal processing (such as DWT) and some for the signal analysis (such as CWT). DWT and other fast transforms are more suited for the signal processing, because of the reductions in data necessary for the execution, which is especially important in realtime signal processing applications. On the other hand, CWT is redundant, which leaves a lot of data for analysis at i.e. higher vision applications.

The presented example shows that all mentioned transforms could have problems in segmenting the motion in a presence of larger waves.

\section{REFERENCES}

Anaraki, M.S., Dong, F., Hirota, K. and Nobuhara, H., (2007), A Multidirectional Multiresolution Transform for Image Representation, 8th International Symposium on Advanced Intelligent Systems ISIS 2007, Sokcho-City, Korea, September 5-8, 2007., available at: http://isis2007.fuzzy.or.kr/submission/upload/A1331.pdf, [accessed 22 August 2011.].

Antoniou, A., (2006), Digital Signal Processing - Signals, Systems and Filters, New York: McGrow-Hill Inc.

Candés, E., Demanet, L., Donoho, D. and Ying, L., (2006), Fast Discrete Curvelet Transforms, Multiscale Modeling and Simulation, 5(3), pp. 861-899., http://dx.doi.org/\%2010.1137/05064182X

Christopher, H. and Walnut, D. F., (2006), Fundamental Papers in Wavelet Theory, London: Princeton University Press.

Crochiere, R. E. and Sambur, M. R., (1977), A Variable-Band Coding Scheme for Speech Encoding at 4.8 kb/s, The Bell System Technical Journal, 56(5), pp. 771-779.

Crochiere, R. E., Webber, S. A. and Flanagan, J. L., (1975), Digital Coding of Speech in Subbands, The Bell System Technical Journal, 55(8), pp. 1069-1085.

Daubechies, I., (1992), Ten Lectures on Wavelets, Philadelphia: Society for Industrial and Applied Mathematics.

Donoho, D., (1999), Wedgelets: Nearly-minimax estimation of edges, Annals of Statistics, 27 (3), pp. 859-897., http://dx.doi.org/10.1109/IEMBS.1995.575241

Donoho, D., Duncan, M. R., Huo, X. and Levi, O., (1999), Wavelab, available at: http:// www-stat.stanford.edu /_wavelab/, [accessed 12 August 2011.]

Führ, H., Demaret, L. and Friedrich, F., (2006), Beyond wavelets: New image representation paradigms. Chapter 7 in book: Document and Image Compression, ed. Barni, M., London: CRC Press.

Gabor, D., (1946), Theory of communication, Journal of the Institute of Electrical Engineers, 93(26), pp. 429-457.

Gagnon, L., Lina, J. M. and Goulard, B., (1995), Sharpening enhancement of digitized mammograms with complex symmetric Daubechies wavelets, IEEE International Conference of Engineering in Medicine and Biology Society, Montréal, Canada, September 20 - 23, 1995., IEEE Press, pp. 543 - 544. http://dx.doi.org/10.1109/IEMBS.1995.575241

Golub, G. H., Loan, V. and Charles, F., (1996), Matrix Computations, (3rd ed.), New York: Johns Hopkins.

Herley, C., Kovačević, J., Ramchandran, K. and Vetterli, M., (1993), Tilings of the Time-Frequency Plane: Construction of Arbitrary Orthogonal Bases and Fast Tiling Algorithms, IEEE Transactions on Signal Processing, 41(12), pp. 3341 - 3359. http://dx.doi.org/10.1109/78.258078

Jansen, M. and Oonincx, P., (2005), Second Generation Wavelets and Applications, London: Springer - Verlag.

Kingsbury, N. G. and Magarey, J. F. A., (1997), Wavelet Transforms in Image Processing, Proc. First European Conference on Signal Analysis and Prediction, Prague, Czech Republic, June 24 - 27, Birkhäuser, pp. 23 - 24., available at: http://www-sigproc. eng.cam.ac.uk/ ngk/publications/ngk97b.zip, [accessed 18 August 2011.].

Kingsbury, N., (1998), The Dual-tree Complex Wavelet Transform: A New Efficient Tool for Image Restoration and Enhancement, Proc. European Signal Processing Conference, EUSIPCO 98, Rhodes, 8-11 September, 1998, University of Athens, pp. $319-322$. 
Lina, J. M. and Gagnon, L., (1995), Image enhancements with symmetric Daubechies' wavelets, SPIE Aerosense: Wavelet Applications in Signal and Image Processing III, 2569(1), pp. 196-207.

Mallat, S., (1999), A Wavelet Tour of Signal Processing, New York: Academic Press.

Mallat, S., (2009), A Wavelet Tour of Signal Processing, 3rd Edition, New York: Academic Press.

Melchior, P., Meneghetti, M. and Bartelmann, M., (2007), Reliable Shapelet Image Analysis, Astronomy and Astrophysics, 463(3), pp. 1215-1225., http://dx.doi.org/10.1051/0004-6361:20066259

Misiti, M., Misiti, Y., Oppenheim, G. and Poggi, J. M., (1997), Wavelet Toolbox - User's Guide, For Use with MATLAB, Version 2, The Mathwork Inc.

Pennec E. and Mallat, S., (2005), Sparse Geometric Image Representations With Bandelets, IEEE Transactions on Image Processing, 14(4), pp. 423-438., http://dx.doi.org/10.1109/TIP.2005.843753

Peyre, G., Pennec, E. and Dossal, C., (2007) Geometric estimation with orthogonal bandlet bases, Proceedings SPIE Wavelets XII, San Diego, CA, August 26 - 29, 2007. SPIE, pp. 67010M.1-67010M.10., available at: http://univ-paris-diderot.academia. edu/ ErwanLePennec/Papers/607768/Geometric_estimation_with_orthogonal_ bandlet_bases, [accessed 18 August 2011.].

Polikar, R., (2002), Lecturers notes, Rowan University, Glassboro, available at: http:// www.engineering.rowan.edu/ polikar/CLASSESS/ECE554, [accessed 12 January 2010.].

Proakis, J. G. and Manolakis, D. K., (2007), Digital Signal Processing, 4th Ed., New York: Prentice-Hall.

Refregier, A., (2003), Shapelets: I. A Method for Image Analysis, Monthly Notices of the Royal Astronomical Society, 338(1), pp. 35-47.

http://dx.doi.org/10.1046/j.1365-8711.2003.05901.x
Rioul, O. and Vetterli, M., (1991), Wavelets and Signal Processing, IEEE Signal Processing Magazine, 8(10), pp. 14-38, http://dx.doi.org/10.1109/ 79.91217

Selesnick, I. W., Baraniuk, R. G. and Kingsbury, N. G., (2005), The Dual-Tree Complex Wavelet Transform, IEEE Signal Processing Magazine, 22(6), pp. 123-151., http://dx.doi.org/10.1109/ MSP.2005.1550194

Strang, G. and Nquyen, T., (1997), Wavelets and Filter Banks, Boston: Wellesly Cambridge Press.

Sweldens, W., (1998), The Lifting scheme: a construction of second generation wavelets, SIAM Journal on Mathematical Analysis, 29(2), pp. 511-546., http://dx.doi.org/10.1137/ S003614109528905

Vetterli, M. and Gall, D. L., (1989), Perfect Reconstruction FIR Filter Banks: Some Properties and Factorizations, IEEE Transactions on Acoustics, Speech, Signal Processing, 37(7), pp. 1057-1071., http://dx.doi.org/10.1109/29.32283

Vetterli, M. and Kovačević, J., (1995), Wavelets and Subband Coding, London: Prentice Hall.

Wichmann, E. H., (1988), Quantum phisics, Textbook of University of Berkeley, Croatian translation, Zagreb: Tehnička knjiga.

Wickerhauser, M. V., (1994), Adapted Wavelet Analysis from Theory to Software, New York: IEEE Press.

Wu, B. and Nevatia, R., (2005), Detection of Multiple, Partially Occluded Humans in a Single Image by Bayesian Combination of Edgelet Part Detectors, 10th IEEE International Conference on Computer Vision, Beijing, China, October 17-20, 2005. Springer, pp. 90-97.,

http://dx.doi.org/ 10.1109/ICCV.2005.74 